\section{Adaptações neuromusculares ao treinamento de força e concorrente em homens idosos}

\section{Neuromuscular adaptations to strength and concurrent training in elderly men}

\author{
Eduardo Lusa Cadore \\ Ronei Silveira Pinto \\ Luiz Fernando Martins Kruel
}

Resumo - O objetivo desse estudo foi revisar os resultados acerca das adaptações neuromusculares ao treinamento de força (TF) e concorrente (TC) em homens idosos. Foram consultadas as bases de dados da Pubmed, Scopus e Scielo de 1980 a 2012. A partir dessa busca, 3390 artigos tiveram seus títulos avaliados e 127 foram selecionados para uma segunda análise para leitura dos abstracts. Destes, 92 artigos foram lidos completamente e 25 artigos foram selecionados e tiveram seus resultados descritos. Diversos estudos demonstraram que idosos submetidos ao TF apresentam aumento na força, potência, ativação e massa muscular. A melhora na força decorrente do TF pode ser explicada através de adaptações neurais e morfológicas. As principais adaptações neurais ao TF consistem no aumento no recrutamento das unidades motoras (UMs), bem como no aumento na freqüência de disparo das UMs. Já as adaptações morfológicas incluem o aumento da área de secção transversa (AST) fisiológica muscular, bem como no aumento na espessura muscular, ângulo de penação das fibras e modificações nas isoformas de cadeia pesada de miosina e conversão de fibras do subtipo IIX para IIa. Recomenda-se a inclusão do TF de intensidade moderada a alta (65-85\% da força máxima) na rotina dessa população para a melhora da função neuromuscular. Embora o TC promova adaptações neuromusculares significativas, a magnitude dessas adaptações pode ser inferior quando comparada ao TF. Apesar de o TC resultar em interferência nas adaptações neuromusculares, o TC também promove melhora na função cardiovascular, sendo essa intervenção mais recomendada para promoção da saúde em idosos.

Palavras-chave: Adaptações neurais; Envelhecimento, Massa muscular; Treinamento físico.

Abstract - This paper aimed to review the results of studies on neuromuscular adaptations to strength training (ST) and concurrent training (CT) in elderly men. A literature search was conducted using PubMed, Scopus, and SciELO. The search was limited to studies published from 1980 to 2012. A total of 3,390 articles were retrieved. After reading their titles, 127 studies were further evaluated by reading their abstracts. This resulted in 92 papers that were read in full; 25 of these were selected and their results were described in the present review. Several studies showed that, in elderly subjects, ST can produce increases in muscle strength, power, activation and mass. ST-induced strength gain may be explained by neural and morphological adaptations. The main neural adaptations to ST included increased recruitment of motor units and increased motor unit firing rate. Morphological adaptations included increases in the physiological cross-sectional area (CSA) of the muscle, in muscle thickness, in muscle fiber pennation angle, and changes in muscle myosin heavy-chain isoforms, resulting in the conversion of muscle fiber from subtype IIx to IIa. The inclusion of moderate-to-high intensity (60-85\% of maximum strength) ST in the routine of this population is recommended to improve neuromuscular function. CT can promote significant neuromuscular adaptations, but these gains may be of a lower magnitude than those obtained with ST. Although CT has an interference effect on neuromuscular adaptations, it also promotes improvement in cardiovascular function and is therefore the most frequently recommended intervention for health promotion in the elderly.

Key words: Aging; Muscle mass; Neural adaptations; Physical training
1 Universidade Federal do Rio Grande do Sul. Escola de Educação Física. Laboratório de Pesquisa do Exercício. Porto Alegre, RS. Brasil

Recebido em 12/09/11 Revisado em 05/01/12 Aprovado em 11/03/12 


\section{INTRODUÇÃO}

Indivíduos idosos submetidos ao treinamento de força (TF) apresentam aumento significativo na força muscular ${ }^{1-4}$, potência muscular ${ }^{5,6}$, ativação muscular ${ }^{7-11}$ e massa muscular ${ }^{12,13}$. A melhora na força muscular decorrente do treinamento de força nessa população aparenta ser semelhante $\mathrm{e}^{14-16}$ do que à observada em indivíduos jovens, o que lhe confere grande treinabilidade, apesar do prejuízo na função neuromuscular decorrente do envelhecimento9.

Já o treino concorrente, que consiste na execução do treinamento de força simultaneamente ao treinamento aeróbio ${ }^{17-20}$, pode resultar em adaptações neuromusculares de magnitude inferior à obtida pelo treino de força isolado. Isso tem sido demonstrado por estudos que encontraram menor aumento na força muscular quando o treino aeróbio foi executado simultaneamente ao treino de força ${ }^{15,17}$. Além disso, estudos comparando os treinos de força e concorrente têm demonstrado diferentes adaptações na ativação $0^{15}$ e massa muscular ${ }^{17}$. No entanto, um reduzido número de estudos comparou as adaptações neuromusculares decorrentes dos treinamentos de força e concorrente em idosos e os seus resultados são controversos e inconcluivos ${ }^{15,19-20}$, com alguns estudos observando adaptações semelhantes entre os tipos de treinamento ${ }^{19-20}$, enquanto outros observaram maior magnitude de adaptação ao treino de força $\mathrm{a}^{15,18} \mathrm{em}$ idosos. Sendo assim, o objetivo desse estudo foi revisar os resultados dos estudos que investigaram as adaptações neuromusculares aos treinamentos de força e concorrente em homens idosos.

\section{PROCEDIMENTOS METODOLÓGICOS}

Para elaboração da presente revisão, foram consultadas as bases de dados da Pubmed, Scopus e Scielo no período de 1980 a 2012, com as seguintes palavras chave: strenght training in elderly, resistance training in elderly, concurrent tranining, neuromuscular adaptations, hypertrophy, bem como as palavras correspondentes em português. A partir dessa busca, 3390 artigos tiveram seus títulos avaliados. Foram selecionados para um segunda análise 127 artigos, que tiveram seus abstracts lidos. Destes, 92 artigos foram lidos na integra e 25 artigos foram selecionados e tiveram seus resultados descritos nessa revisão. Os critérios gerais de inclusão foram baseados nas recomendações de Campbell e Stanley ${ }^{21}$, que incluíram: (a) estudos randomizados; (b) estudos utilizando instrumentos com alta reprodutibilidade e validade; (c) estudos com mínima perda amostral; (d) estudos com a metodologia de treinamento de força, concorrente ou ambos detalhada; e, (e) estudos nos quais os testes de força, bem como os mecanismos de aumento de força (i.e., neurais e/ou morfológicos) foram avaliados antes e depois do período de treinamento. Especificamente com relação ao treino de força, foram selecionados os artigos sobre treinamento de força em idosos que submeteram homens saudáveis de 60 anos ou mais a diferentes períodos do treinamento de força, avaliaram modificações 
decorrentes do treinamento na força muscular dinâmica ou isométrica, na ativação muscular através do sinal eletromiográfico e na massa muscular através de métodos de imagem como ressonância magnética, ultrasonografia, tomografia computadorizada ou biópsia muscular. Especificamente em relação ao treinamento concorrente, foram selecionados os artigos que investigaram esse tipo de intervenção em idosos saudáveis de 60 anos ou mais a diferentes períodos do treinamento, estabelecendo uma comparação entre as adaptações ao treinamento de força e concorrente.

\section{TREINAMENTO DE FORÇA EM IDOSOS}

O treinamento de força (TF) é uma intervenção efetiva para o aumento na força $^{1-3,6}$, potência ${ }^{6,13,21}$, ativaçãa $0^{7,8,10,13,15}$ e massa muscular ${ }^{2,6,8,11,16}$. Adaptações na função neuromuscular decorrente do treinamento de força nessa população aparenta ser semelhante do que em indivíduos jovens ${ }^{16}$, e essas adaptações resultam em melhora significativa na capacidade funcional dessa população ${ }^{22}$.

\section{Efeitos na produção de força e potência muscular}

No Quadro 1 são apresentados as características do programa de treinamento e os resultados de alguns estudos que investigaram os efeitos do treino de força na função neuromuscular em homens idosos.

Os resultados sugerem que estudos utilizando 2 - 6 séries (aumento de volume progressivo durante a periodização), com intensidades variando de 40 a $80 \%$ da força máxima (i.e., 1 repetição máxima - 1RM), com 2 sessões semanais em períodos de 12 a 24 semanas, observaram incrementos médios na força muscular dinâmica de $30 \%{ }^{10-13,23}$, bem como na força muscular isométrica de $10-60 \%^{7,10-13}$. Maiores incrementos na força muscular dinâmica podem ser observados com o aumento do número de sessões semanais ${ }^{15}$. Em estudo de Cadore et al. ${ }^{15}$, foi observado aumento de $67 \%$ na força dinâmica máxima em homens idosos que treinaram 3 vezes por semana durante 12 semanas, o qual o volume/intensidade inicial foi de 20 repetições máximas (RM), sendo de 6-8 RM na fase final do período de treino.

Um aspecto que influencia as adaptações neuromusculares ao TF em homens idosos é a especificidade das ações musculares. Em estudo de Häkkinen et al. ${ }^{8}$, homens idosos foram divididos em grupos de treino unilateral e bilateral, sendo ambos avaliados na força máxima bilateral e unilateral. Foram observados aumentos na força bilateral do quadríceps de todos os indivíduos, com maior aumento relativo para o grupo que treinou bilateralmente (19 vs. 13\%), bem como na força unilateral, sendo maior aumento relativo para o grupo que treinou unilateralmente (17 vs. $10 \%$ ).

Cabe salientar, relativamente ao método de treino utilizado, a importância da execução de parte das séries com ações musculares explosivas durante o treinamento, visando modificações relacionadas à produção de força rápida em indivíduos idosos, já que variáveis relacionadas à potência muscular ou à taxa de produção de força possuem estreita relação com a 
capacidade funcional em idosos ${ }^{2,22}$. Em estudos de Bottaro et al. ${ }^{22}$ e Nogueira et al. ${ }^{6}$, foram observados incrementos significativos na produção de força rápida nos idosos que treinaram em alta velocidade de execução e nenhum incremento nessa capacidade nos idosos que treinaram somente com ações musculares lentas.

Os resultados dos estudos supracitados demonstram claramente a capacidade de aumento na força muscular em indivíduos idosos ${ }^{6,12-15}$. Diferenças na magnitude de aumento na força nos diferentes estudos podem ter ocorrido devido a fatores como condicionamento físico inicial da amostra, tempo de treinamento e intensidade fisiológica das séries (repetições máximas vs. repetições submáximas). A importância do treinamento de força e a consistência de seus efeitos nessa população podem ser observadas, já que mesmo após períodos de destreino, a força muscular ainda permanece maior do que a registrada anteriormente ao período de treinamento ${ }^{12}$.

\section{Mecanismos fisiológicos responsáveis pelo aumento na força muscular}

A melhora na força muscular decorrente do TF pode ser explicada a partir de adaptações neurais e morfológicas. As principais adaptações neurais ao TF consistem no aumento na capacidade de recrutamento nas unidades motoras, bem como no aumento na freqüência de disparo dessas unidades motoras ${ }^{5,7}$. Entre os métodos de avaliação das adaptações neurais ao treino de força, o mais comum é a quantificação da amplitude do sinal eletromiográfico $(\mathrm{EMG})^{5,7,17}$, já que um aumento nas unidades motoras recrutadas, bem como na sua freqüência de disparo resultam em aumento na amplitude desse sinal $1^{9-15}$. Outras adaptações neurais sugeridas para explicar o aumento da força máxima são a redução da coativação dos músculos antagonistas ${ }^{11}$, além da inibição da ativação do órgão tendinoso de Golgi durante a produção de tensão máxima do músculo. Ainda, um maior sincronismo de fibras musculares, embora não seja sugerido como fator importante para a melhora da força máxima, resulta em um menor tempo de obtenção da força máxima, sendo, portanto, um mecanismo responsável pelo aumento na taxa de produção de força ${ }^{9-11}$.

Já as adaptações morfológicas ao TF incluem o aumento da área de secção transversa fisiológica (AST) da fibra muscular ${ }^{17,24}$, o que resulta no aumento na AST do músculo ${ }^{13}$, bem como no aumento na espessura muscular $^{6}$ e uma modificação entre as isoformas de cadeia pesada de miosina, o que ocorre a partir de uma conversão de fibras do subtipo IIX para o subtipo IIa ${ }^{17,24}$. Modificações na AST da fibra são comumente avaliadas a partir de técnicas histoquímicas realizadas após biópsia muscular ${ }^{17}$, enquanto modificações na AST total do músculo e na espessura muscular são avaliadas a partir de técnicas de imagem como ressonância magnética ${ }^{23}$, tomografia computadorizada ${ }^{9}$ ou ultrasonografia ${ }^{6,13}$.

\section{Efeitos na ativação muscular}

Diversos estudos têm demonstrado aumento da amplitude do sinal EMG paralelamente ao aumento de força observado ${ }^{7,8,12,15}$, geralmente investi- 
gando a amplitude do sinal eletromiográfico (EMG) dos músculos vasto lateral, vasto medial e reto da coxa, como indicador da ativação muscular do quadríceps em homens idosos.

Os incrementos no sinal EMG são mais proeminentes nas primeiras 4 a 8 semanas, fase de treinamento em que ocorrem preferencialmente adaptações neurais ${ }^{7,8,10}$. Da mesma forma que o aumento na força muscular, adaptações no sinal EMG seguem um padrão de especificidade, com maior aumento no sinal EMG em contrações bilaterais observados como adaptação ao treino bilateral (19 vs. $10 \%$ ), bem como maior aumento em contrações unilaterais observados como adaptação ao treino unilateral (9 vs. $7 \%)^{8}$. Ainda, uma estreita relação pode ser observada entre os incrementos na força muscular e na magnitude do sinal EMG, evidenciando a importância das adaptações neurais nas primeiras semanas de treinamento $(\mathrm{r}=0,59-0,65 ; \mathrm{P}<0,05)^{8,12}$.

Uma variável relacionada ao sinal EMG, importante na avaliação das adaptações neurais ao TF, é a taxa de aumento do sinal EMG em determinados intervalos (i.e., $100 \mathrm{~ms}, 500 \mathrm{~ms}$ ), o que evidencia uma melhora na capacidade de recrutamento rápido de um grupo muscular. Contudo, a exemplo da taxa de produção de força, essas variáveis aparentam ser incrementadas somente quando ações musculares explosivas são agregadas ao treino ${ }^{10}$.

Outro adaptação neural decorrente do TF é a melhora na economia neuromuscular ${ }^{15,18,25}$. Em estudo prévio do nosso laboratório, Cadore et al. ${ }^{15}$ observaram uma redução no sinal EMG normalizado a 40, 60 e $80 \%$ da força máxima isométrica (CIVM) pré treinamento no vasto lateral e 60 e $80 \%$ no reto da coxa, após o TF em indivíduos idosos. Esses resultados sugerem que para a mesma carga absoluta, os indivíduos necessitaram de um menor número de unidades motoras após o treinamento.

Com base nos resultados dos estudos supracitados pode-se inferir que o treinamento de força resulta em um aumento na amplitude do sinal EMG máximo em homens idosos ${ }^{7,8,13,15,23}$, bem como numa redução no sinal EMG normalizado para a mesma carga submáxima (Quadro 1). Esse aumento está relacionado com adaptações neurais ao treinamento de força, já que esse aumento ocorre paralelamente ao aumento na força máxima ${ }^{7-15}$.

\section{Efeitos na massa muscular}

Diversos estudos têm demonstrado incrementos importantes na massa muscular em indivíduos idosos. Embora a capacidade de aumento na massa muscular nessa população possa ser menor quando comparada a de indivíduos jovens ${ }^{16}$, estudos têm demonstrado aumentos entre 5 e $14 \%$ da AST e espessura muscular do quadríceps femoral nessa população em períodos de treinamento que variam de 10 a 24 semanas s-8,23. $^{6}$.

Os resultados dos estudos revisados sugerem que existe uma preservação do potencial de hipertrofia muscular em indivíduos idosos, embora esse potencial seja inferior ao observado em indivíduos joven ${ }^{16}$, diferentemente do que ocorre com potencial de aumento na força muscular (semelhante 
entre jovens e idosos). Essa hipertrofia pode ser observada mesmo em estudos com TF de curta duração (i.e., 10 semanas) ${ }^{16,23}$, com periodização linear ${ }^{7}$ ou ondulatória ${ }^{16}$, freqüência semanal de duas ${ }^{6}$ a três sessões por semana ${ }^{23}$, cargas moderadas a altas (60 a $90 \%$ de 1 RM) e múltiplas séries $^{12,16,23}$ (Quadro 1).

Quadro 1. Adaptações neuromusculares ao treinamento de força.

\begin{tabular}{|c|c|c|c|}
\hline Autor & $\begin{array}{l}\text { Período e } \\
\text { Frequência }\end{array}$ & Volume e intensidade & Resultados \\
\hline Häkkinen e Häkkinen ${ }^{7}$ & 12 sem; $2 x /$ sem & $\begin{array}{l}2-5 \text { séries de } 3-15 \text { repetições } \\
\text { ( } 30-90 \% \text { de } 1 \mathrm{RM}) 30-80 \% \text { de } 1 \mathrm{RM} . \\
\text { Contrações lentas e explosivas. }\end{array}$ & $\begin{array}{l}\uparrow P T(20 \%)^{* *} ; \uparrow E M G \text { VL, VM e RC }(\sim 20 \%)^{*} ; \uparrow A S T \\
\text { QF }(9 \%)^{* *} \text {. }\end{array}$ \\
\hline Häkkinen et al. ${ }^{8}$ & 12 sem; $2 x /$ sem & $\begin{array}{l}\text { 2- } 6 \text { séries de } 8-15 \text { repetições } \\
\text { ( } 40-90 \% \text { de } 1 \mathrm{RM}) \text { unilateral (UNI) e } \\
\text { bilateral (BIL). Contrações lentas e } \\
\text { explosivas. }\end{array}$ & 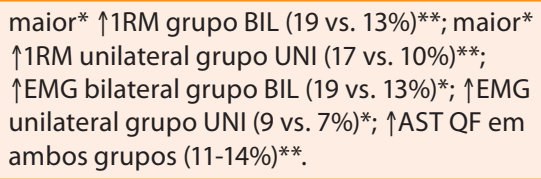 \\
\hline Häkkinen et al. ${ }^{10}$ & 24 sem; $2 x /$ sem & $\begin{array}{l}\text { 3-5 séries de } 3-15 \text { repetições ( } 50- \\
90 \% \text { de } 1 \text { RM). Contrações lentas e } \\
\text { explosivas }(20 \%) \text {. }\end{array}$ & $\begin{array}{l}\uparrow 1 \mathrm{RM}(21 \%)^{* *} ; \uparrow P \mathrm{PT}(36 \%)^{* * *} ; \uparrow \operatorname{TPF}(40 \%)^{*} ; \uparrow S J \\
(24 \%)^{* * *} ; \uparrow \mathrm{EMG}^{*} \text { VL e VM Sem } \uparrow \text { AST QF }(2 \%, \\
\text { NS). }\end{array}$ \\
\hline Kraemer et al. ${ }^{16}$ & 10 sem; $3 x /$ sem & $\begin{array}{l}\text { periodização ondulatória: 2-5 } \\
\text { séries de 3-5RM; 8-10RM e 12- } \\
\text { 15RM. }\end{array}$ & $\uparrow 1 \mathrm{RM}(10 \%)^{*} ; \uparrow A S T$ QF $(6 \%)^{*}$. \\
\hline Häkkinen et al..11 & 10 sem; $2 x /$ sem & $\begin{array}{l}\text { 3-5 séries de 3-15 repetições ( } 50 \text { - } \\
90 \% \text { de } 1 \text { RM). Contrações lentas e } \\
\text { explosivas (20\%). }\end{array}$ & $\begin{array}{l}\uparrow P T(16 \%)^{* *} ; \uparrow E M G * \text { VL e VM; } \uparrow A S T \text { QF } \\
(8,5 \%)^{* * *} ; \uparrow \mathrm{AST}^{*} \text { fibras I e II. }\end{array}$ \\
\hline Häkkinen et al. ${ }^{12}$ & 24 sem; $2 x /$ sem & $\begin{array}{l}\text { 3-6 séries de } 6-15 \text { repetições ( } 50- \\
80 \% \text { de } 1 \text { RM). Contrações lentas e } \\
\text { explosivas. }\end{array}$ & $\begin{array}{l}\uparrow 1 \mathrm{RM}(29 \%)^{* * *} ; \uparrow \mathrm{EMG}^{*} \text { VL e VM; } \uparrow \text { SJ }(22 \%)^{* * *} ; \\
\uparrow \text { AST QF }(7 \%)^{* * *} \text {. }\end{array}$ \\
\hline Häkkinen et al. ${ }^{13}$ & 24 sem; $2 x /$ sem & $\begin{array}{l}\text { 3-5 séries de } 6-15 \text { repetições ( } 50- \\
80 \% \text { de } 1 \text { RM). Contrações lentas e } \\
\text { explosivas. }\end{array}$ & $\begin{array}{l}\uparrow P T(36 \%)^{* * * *} ; \uparrow E M G * \text { VL e VM; } \uparrow \text { TPF }(40 \%)^{*} ; \\
\uparrow 1 R M(21 \%)^{* * * *} ; \text { Sem modificações na AST } \\
\text { fibras I e II. }\end{array}$ \\
\hline Izquierdo et al. ${ }^{2}$ & 16 sem; $2 x / s e m$ & $\begin{array}{l}\text { 3-5 séries de 6-15 repetições ( } 50 \text { - } \\
80 \% \text { de } 1 \mathrm{RM}) \text {. Contrações lentas e } \\
\text { explosivas. }\end{array}$ & $\begin{array}{l}\uparrow 1 \mathrm{RM}(25-41 \%)^{* * *} ; \uparrow \mathrm{PT}(26 \%)^{* * *} ; \uparrow \text { potência } \\
\text { de } 20 \text { a } 80 \% \text { de } 1 \mathrm{RM}(15-60 \%)^{* *} ; \uparrow \text { AST QF } \\
(11 \%)^{* *}\end{array}$ \\
\hline Häkkinen et al. ${ }^{1}$ & 24 sem; $2 x /$ sem & $\begin{array}{l}\text { 3-5 séries de 6-15 repetições (50- } \\
80 \% \text { de } 1 \text { RM). Contrações lentas e } \\
\text { explosivas (20\%). }\end{array}$ & $\uparrow 1 \mathrm{RM}(21 \%)^{* * *} ; \uparrow A S T^{*}$ fibras I, Ila e IIX. \\
\hline Bottaro et al. ${ }^{22}$ & 10 sem; $2 x /$ sem & $\begin{array}{l}3 \text { séries de } 8 \text { a } 10 \text { repetições }(40 \text { a } \\
60 \% \text { de } 1 \text { RM); contrações lentas } \\
(C L) \text { vs. explosivas (CE) }\end{array}$ & $\begin{array}{l}\uparrow 1 \mathrm{RM}(25 \%)^{*} \text { nos } 2 \text { grupos; } \uparrow \text { potência a } \\
60 \% \text { d } 1 \mathrm{RM} \text {, maior em CE ( } 31 \text { vs. } 8 \%)^{*} \text {. Maior } \\
\text { aumento em CE também observados nos } \\
\text { testes } 8 \mathrm{ft} \text {-up-and-go ( } 15 \text { vs. } 0.8 \%)^{*} \text { e } 30 \text { s } \\
\text { chair stand }(43 \text { vs. } 6 \%)^{*}\end{array}$ \\
\hline Cannon et al. ${ }^{23}$ & 10 sem; $2 x /$ sem & $\begin{array}{l}3 \text { séries de } 10 \text { repetições } \\
75 \% \text { de } 1 \mathrm{RM}) \text {. }\end{array}$ & $\begin{array}{l}\text { } P T(18 \%)^{*} ; \uparrow E M G * \text { VL e VM }(21 \%)^{*} ; \uparrow A S T \text { QF } \\
(11 \%)^{*} \text {. }\end{array}$ \\
\hline Slivka et al. ${ }^{3}$ & 12 sem; $3 x /$ sem & $\begin{array}{l}3 \text { séries de } 10 \text { repetições } \quad \text { ( } 70 \% \\
\text { de } 1 \mathrm{RM}) \text {. }\end{array}$ & $\uparrow 1 \mathrm{RM}(41 \%)^{*} ;$ AST QF $(2 \%)^{*}$ \\
\hline Nogueira et al. ${ }^{6}$ & 10 sem; $2 x /$ sem & $\begin{array}{l}3 \text { séries de } 8 \text { a } 10 \text { repetições }(40 \text { a } \\
60 \% \text { de } 1 \mathrm{RM}) ; \text { contrações lentas } \\
(\mathrm{CL}) \text { vs. explosivas (CE) }\end{array}$ & $\begin{array}{l}\uparrow 1 \mathrm{RM}(25 \%)^{*} \text { nos } 2 \text { grupos; } \uparrow \text { espessura do RC } \\
\text { somente em CE }(11 \%)^{*} \text { e do BIC em CE }(14 \%)^{*} \\
\text { e CL }(6,7 \%)^{*} \text {. }\end{array}$ \\
\hline Raj et al. ${ }^{4}$ & 16 sem; $2 x /$ sem & $\begin{array}{l}\text { TF } 2 \text { séries de } 10 \text { repetições ( } 75 \% \\
1 \text { RM) vs. } 3 \text { séries de } 10 \text { repetições } \\
(50 \% 1 \text { RM) com a fase excêntrica } \\
\text { realizada UNI. }\end{array}$ & $\begin{array}{l}\uparrow 1 \mathrm{RM}(23 \%)^{* *} \text { nos } 2 \text { grupos; } \uparrow \text { espessura do } \\
\text { VL somente no grupo com ênfase excêntrica } \\
\text { UNI }(5 \%)^{*} \text {. }\end{array}$ \\
\hline
\end{tabular}

$\uparrow$, aumento; min, minutos; 1RM, 1 repetição máxima; PT, pico de torque isométrico; SJ, squat jump; AST, área de secção transversal; QF, quadríceps femoral; VL, vasto lateral; VM, vasto medial; RC, reto da coxa; BIC, bíceps braquial; sem, semanas; $\mathrm{X} / \mathrm{sem}$, número de treinos por semana; TF, treinamento de força; EMG, sinal eletromiográfico; TPF, taxa máxima de produção de força; ${ }^{*} P<0,05$; ${ }^{* * P}<0,01 ;{ }^{* * * P}<0,001$; NS, não significativo. 


\section{TREINAMENTO CONCORRENTE EM IDOSOS}

A literatura nos mostra resultados controversos a respeito dos efeitos do treino aeróbio (TA) realizado simultaneamente ao TF nos ganhos de força e potência muscular em diversas populações (homens ou mulheres, jovens ou idosos, atletas ou sedentários). Alguns estudos demonstraram que o TA pode reduzir o ganho de força e potência decorrentes do TF quando ambos os tipos de treino são executados simultaneamente (i.e., treinamento concorrente) e esse efeito têm sido denominado de "efeito de interferência"17,26-28. Por outro lado, diversos outros estudos observaram ganhos semelhantes na força muscular comparando o TF com o treino concorrente (TC ${ }^{19,20,29-31}$. Apesar de diversos estudos terem sido realizados investigando indivíduos jovens, poucos investigaram o desempenho da força e capacidade aeróbica do treino concorrente em indivíduos ido$\operatorname{sos}^{19,20,29,32,33}$. A vantagem da prescrição do treinamento concorrente, é que mesmo que ocorra interferência nos ganhos de força ou potência, esse tipo de treinamento apresenta os mesmos ganhos de capacidade aeróbica que o treino aeróbico isolado ${ }^{17,18,27,29}$.

\section{Efeitos na força muscular}

Na Quadro 2 são apresentados as características do programa de treinamento e os resultados de alguns estudos que compararam os efeitos do treino de força e concorrente na função neuromuscular em homens idosos.

Em estudo realizado por Wood et al. ${ }^{29}$, indivíduos idosos foram submetidos ao TF e TC durante 12 semanas, com o grupo de TF executando 2 séries e o grupo de TC executando apenas 1 série. Esses autores observaram aumento semelhante na força muscular em ambos os grupos (38-44\%). Em outro estudo, Izquierdo et al. ${ }^{19}$ investigaram homens idosos após 16 semanas de treinamento, com o grupo de TF executando 2 sessões por semana e o grupo de TC executando uma sessão por semana de TF e outra de TA em cicloergômetro. Não foram observadas diferenças nas adaptações na força muscular entre o TF e o TC, sugerindo um efeito sinérgico do TA no ciclo ergômetro com o TF (grupo TC), o que resultou nos mesmos ganhos comparados ao TF. Contudo, nos estudos de Wood et al. ${ }^{29}$ e Izquierdo et al. ${ }^{19}$, os grupos de TC treinaram menos séries por exercício do que os grupos de TF, o que dificulta comparações dos ganhos entre TF e TC.

Utilizando o mesmo volume de treinamento para os grupos de TF e TC durante 21 semanas com frequência semanal de 2 vezes por semana, volume variando de 2 - 4 séries e cargas de 40 a $80 \%$ de 1RM, Karavirta et al. ${ }^{20}$ observaram ganhos similares de força dinâmica e isométrica entre os grupos. Em outros estudos utilizando periodizações de treinamento e frequência semanal similares ${ }^{34-37}$ ganhos semelhantes de força foram observados entre o TF e TC.

Já em estudos no nosso Laboratório, Cadore et al. ${ }^{15}$, observaram um ganhos de força dinâmica superiores no grupo de força (67\%) compa- 
rado ao grupo concorrente (41\%) após 12 semanas de treinamento, o que não foi observado em exercício para membros superiores (31 vs. $33 \%$, respectivamente), sugerindo que o TA interferiu negativamente somente quando o mesmo grupo muscular foi envolvido em ambos tipos de treinamento. Cabe salientar, não obstante, que mesmo encontrando interferência do TA nos ganhos do TF, ainda assim os incrementos na força muscular observados no grupo de TC nesse estudo foram semelhantes ou superiores aos ganhos de força observados nos estudos investigando idosos supracitados: $21-44 \%^{20,29,35-37}$, o que pode ser justificado pela maior frequência semanal (3 vezes por semana). Nesse estudo foi especulado que a fatiga resultante do TA tenha sido o fator responsável pelo efeito de interferência observado, já que o TA foi sempre executado antes do $\mathrm{TF}^{15}$. Sendo assim, em outro estudo Cadore et al. ${ }^{32}$ investigaram o efeito da ordem do treinamento nas adaptações neuromusculares ao TC em idosos e observaram um ganho de força $50 \%$ superior no grupo que executava o TF antes do TA (35 vs. $22 \%$; $\mathrm{P}<0,01)$. Esses resultados sugerem que com uma maior frequência semanal, os ganhos neuromusculares podem ser otimizados quando o TF é executado antes do TA.

Baseados nos resultados observados com relação ao treinamento concorrente em idosos, pode-se inferir que: (I) realizando o mínimo de frequência semanal de treinamento ( 1 vez por semana $\mathrm{TF}+1 \mathrm{vez}$ por semana TA), o TA executado em ciclo ergômetro pode ter um efeito sinérgico com o TF ${ }^{19}$; (II) frequência semanal de 2 vezes por semana não resulta em efeito de interferência ${ }^{33-37}$; (III) frequência semanal de 3 vezes por semana otimiza os ganhos de força mas resulta em interferência ${ }^{15}$; e, (IV) a interferência observada com a frequência semanal de 3 vezes por semana é relacionada a fatiga resultante do TA quando esse é realizado imediatamente antes do TF e a manipulação da ordem dos exercícios pode prevenir o efeito de interferência quando o TF antecedo o TA ${ }^{32}$.

Entre os mecanismos apontados como responsáveis pela interferência do TA no desenvolvimento da força muscular decorrente do TF estão a interferência nas adaptações neurais, o baixo conteúdo de glicogênio e consequente estado catabólico crônico levando ao overtraining e a interferência no turnover protéico das fibras utilizadas em ambos os tipos de treinamento, o que induz a uma menor magnitude de hipertrofia muscular, especialmente nas fibras do tipo I e à fadiga ocasionada pelo treino aeróbico anterior ao treinamento de força, comprometendo a performance no mesmo ${ }^{38}$.

\section{Efeitos na ativação muscular}

Poucos estudos investigaram as adaptações no sinal EMG decorrentes do treino concorrente e, em indivíduos jovens, nenhuma diferença nas adaptações no sinal EMG máximo tem sido observada entre os treinos de força e concorrente ${ }^{30}$, embora interferência tenha sido observada nos ganhos na ativação muscular rápida ${ }^{39}$. Por outro lado em indivíduos idosos, apesar de 
resultados controversos, um prejuízo nas adaptações neurais parece explicar o efeito de interferência quando o mesmo ocorre nessa população ${ }^{15,32}$.

Investigando indivíduos idosos, Karavirta et al. ${ }^{20}$ demonstraram ganhos na EMG máxima significativamente maiores no grupo de TF comparado ao TC nos músculos vasto lateral e vasto medial após 10 semanas (41 vs $18 \%$ e 35 vs. $11 \%$, respectivamente), ao passo que após 21 semanas as diferenças não se mantiveram, sendo observado o mesmo incremento nesses dois grupos. Da mesma forma que os valores de força supracitados, em outros estudos que utilizaram periodizações de treinamento e frequência semanal similares nenhuma diferença nas adaptações neurais foi observada entre os grupos de $\mathrm{TF}$ e $\mathrm{TC}^{33-37}$.

Já em estudo de Cadore et al. ${ }^{15}$ foi observado aumento significativo na amplitude do sinal EMG somente em resposta ao TF isolado (33\%), sendo que esse aumento foi significativamente maior do que o observado após o TC (16\%). Cabe salientar que essas diferenças ocorreram em paralelo com as diferença nos ganhos de força dinâmica citados anteriormente (67 vs. $41 \%$ em TF e TC, respectivamente). Esses resultados sugerem que o efeito de interferência ocorreu devido a um prejuízo promovido pelo TA nas adaptações neurais ao TF. Já Cadore et al. ${ }^{32}$ observaram maior aumento força por unidade de massa muscular (i.e., qualidade muscular ou tensão específica) nos indivíduos que treinaram o TF antes do TA, comparado com a ordem inversa ( 27 vs. $15 \%, \mathrm{P}<0,01)$. Sendo a qualidade muscular uma medida que estima a contribuição de fatores neurais relacionados com ganhos de força ${ }^{40}$, pode ser sugerido que os maiores ganhos na força musculares obtidos na ordem TF-TA comparado a ordem inversa podem ter ocorrido por diferença nas adaptações neurais.

\section{Efeitos na massa muscular}

Estudos investigando adaptações morfológicas ao treino concorrente em idades mais avançadas são escassos. Izquierdo et al. ${ }^{19}$ investigando homens idosos, não observaram diferenças na hipertrofia muscular após 12 semanas de treino de força ( 2 vezes por semana) e concorrente (um vez de treino de força e outra aeróbio) (ambos 11\% de aumento).

Já em estudo de Karavirta et al. ${ }^{20}$, foi observado um aumento na AST das fibras tipo II do vasto lateral somente no grupo de TF (16\%), ao passo que nenhuma modificação foi observada no grupo de TC nesse estudo. Curiosamente, nesse estudo não foram observadas diferenças na magnitude de incremento nas variáveis de força muscular. Em outro estudo, Cadore et al. ${ }^{32}$ não observaram diferenças nos ganhos de espessura muscular entre grupos executando o TC com diferentes ordens de exercício, mesmo tendo observado diferenças nos ganhos de força.

Com base nos resultados descritos, é possível sugerir que o componente morfológico associado à produção de força não possui relação com o efeito de interferência quando o mesmo ocorre em homens idosos ${ }^{15,32}$. 
Quadro 2. Adaptações neuromusculares ao treinamento concorrente vs. treinamento de força.

\begin{tabular}{|c|c|c|c|}
\hline Autor & Período e Frequência & Volume e intensidade & Resultados \\
\hline Wood et al. ${ }^{29}$ & $\begin{array}{l}12 \text { sem; TF: } 3 x / \text { sem; TA: } \\
\text { 2x/sem: TC: } T F+T A\end{array}$ & $\begin{array}{l}\text { TF: } 2 \text { séries de } 12-15 \text { repetições (75\% } \\
\text { de } 5 \mathrm{RM} \text { ) até } 8-12 \mathrm{RM} \text {. TA: } 60-70 \% \text { da } \\
\mathrm{FC}_{\text {máx }} \text { estimada, } 21-45 \text { min em cicloer- } \\
\text { gômetro. TC: } 1 \text { série de } 8-12 \mathrm{RM}+30 \\
\text { min em cicloergômetro (TA) }\end{array}$ & $\begin{array}{l}\uparrow 1 \mathrm{RM}(15-29 \%)^{*} \text { em TF e TC. } \uparrow \\
\text { performance em testes funcionais } \\
\text { em ambos os grupos*. Nenhuma } \\
\text { diferença entre TF e TC. }\end{array}$ \\
\hline Izquierdo et al. ${ }^{19}$ & $\begin{array}{l}16 \text { sem; TF: } 2 x / \text { sem; TA: } \\
\text { 2x/sem; TC: } 1 x / \text { sem TF + } \\
\text { 1x/sem TA. }\end{array}$ & $\begin{array}{l}\text { 3-5 séries de 6-15 repetições ( } 50-80 \% \\
\text { de } 1 \text { RM) Contrações lentas e explosivas } \\
\text { ( } 20 \% \text { do volume de } 30 \text { a } 50 \% \text { de } 1 \mathrm{RM}) \text {. } \\
\text { TA: } 30 \text { a } 40 \text { min em cicloergômetro, nas } \\
\text { cargas (W) de } 2,3 \text { e } 4 \mathrm{mmol} \mathrm{L}^{-1} \text {. }\end{array}$ & $\begin{array}{l}\uparrow 1 \mathrm{RM} \text { em TF e TC }(22-41 \%)^{* *} ; \uparrow \\
\text { potência muscular a } 45 \% \text { de } 1 \text { RM em } \\
\text { TF e TC }(45 \%)^{*} . \uparrow A S T \text { QF em TF e TC } \\
(\text { ambos } 11 \%)^{*} \text {. Nenhuma diferença } \\
\text { entre TF e TC. }\end{array}$ \\
\hline Sillanpaä et al. ${ }^{33}$ & $\begin{array}{l}21 \text { sem; TF: } 2 x / \text { sem; TA: } \\
\text { 2x/sem; TC: TF + TA } \\
\text { dias alternados. }\end{array}$ & $\begin{array}{l}\text { TF: múltiplas séries (40-90\% de 1RM). } \\
\text { TA: } 30 \text { a } 60 \text { min em cicloergômetro, } \\
\text { intensidades abaixo do } L_{1} \text {, entre } L_{1} \text { e } \\
\mathrm{LV}_{2} \text { e acima do } \mathrm{LV}_{2} .\end{array}$ & $\begin{array}{l}\uparrow 1 \mathrm{RM} \text { em TF e TC }(22 \%)^{* * *} ; \uparrow \text { es- } \\
\text { pessura VL+VM em TF e TC }(9 \%)^{* * *} \text {. } \\
\text { Nenhuma diferença entre TF e TC. }\end{array}$ \\
\hline Sillanpaä et al. ${ }^{34}$ & $\begin{array}{l}21 \text { sem; TF: } 2 x / \text { sem; TA: } \\
\text { 2x/sem; TC: TF + TA dias } \\
\text { alternados. }\end{array}$ & $\begin{array}{l}\text { TF: múltiplas séries (40-90\% de 1RM). } \\
\text { TA: } 30 \text { a } 60 \text { min em cicloergômetro, } \\
\text { intensidades abaixo do } L_{1} \text {, entre } L_{1} \text { e } \\
L_{2} \text { e acima do } L_{2} \text {. }\end{array}$ & $\begin{array}{l}\uparrow P T \text { em TF e TC }(15-17 \%)^{* * *} \text {. Nenhu- } \\
\text { ma diferença entre TF e TC. }\end{array}$ \\
\hline Karavirta et al. ${ }^{37}$ & $\begin{array}{l}21 \text { sem; TF: } 2 x / \text { sem; TA: } \\
\text { 2x/sem; TC: TF + TA, } \\
\text { dias alternados. }\end{array}$ & $\begin{array}{l}\text { TF: múltiplas séries (40-90\% de 1RM). } \\
\text { TA: } 30 \text { a } 60 \text { min em cicloergômetro, } \\
\text { intensidades abaixo do } L_{1} \text {, entre } L_{1} \text { e } \\
\mathrm{LV}_{2} \text { e acima do } \mathrm{LV}_{2} .\end{array}$ & $\begin{array}{l}\uparrow 1 \mathrm{RM} \text { em TF e TC }(22 \%)^{* * *} \text {. Nenhuma } \\
\text { diferença entre TF e TC. }\end{array}$ \\
\hline Cadore et al..$^{15}$ & $\begin{array}{l}12 \text { sem; TF: 3x/sem; TA: } \\
\text { 3x/sem: TC: TF+TA, TA } \\
\text { antes de TF, mesma } \\
\text { sessão. }\end{array}$ & $\begin{array}{l}\text { TF: } 18-20 R M \text { a 6-8RM. TA: } 30 \text { min em } \\
\text { cicloergômetro, } 80 \text { a } 100 \% \text { do } \text { LV }_{2} \text {. }\end{array}$ & $\begin{array}{l}\text { maior } \uparrow 1 \mathrm{RM} \text { em TF }(67 \%)^{* * *} \text { compa- } \\
\text { rado a TC(41\%)***; maior } \uparrow \text { no PT em } \\
\text { TF }(14 \%)^{*} \text { comparado a TC }(1 \%, N S) \text {. } \\
\text { maior } \uparrow \text { EMG VL e RC em TF }(30 \%)^{*} \\
\text { comparado a TC }(16 \%, \text { NS); maior } \uparrow \\
\text { ECO neuromuscular* em TF compa- } \\
\text { rada a TC. }\end{array}$ \\
\hline Holviala et al. ${ }^{35}$ & $\begin{array}{l}21 \text { sem; TF: } 2 x / \text { sem; TA: } \\
\text { 2x/sem; TC: TF + TA, } \\
\text { dias alternados. }\end{array}$ & $\begin{array}{l}\text { TF: múltiplas séries ( } 40-90 \% \text { de } 1 \mathrm{RM}) \text {. } \\
\text { TA: } 30 \text { a } 60 \text { min em cicloergômetro, } \\
\text { intensidades abaixo do } \mathrm{LV}_{1} \text {, entre } \mathrm{LV}_{1} \mathrm{e} \\
\mathrm{LV}_{2} \text { e acima do } \mathrm{LV}_{2} .\end{array}$ & $\begin{array}{l}\uparrow 1 \mathrm{RM} \text { em TF e TC }(22 \%)^{* * *} \text {. Nenhuma } \\
\text { diferença entre TF e TC. } \uparrow \text { EMG VL } \\
(18 \%)^{*} \text { e RF }(14 \%)^{*} \text { em TC e } \uparrow E M G \\
\text { VM }(32 \%) \text { em TF*. } \uparrow P T \text { em TF e TC } \\
(8-12 \%)^{*} \text {. Nenhuma diferença entre } \\
\text { TF e TC. }\end{array}$ \\
\hline Holviala et al. ${ }^{36}$ & $\begin{array}{l}21 \text { sem; TF: } 2 x / \text { sem; TA: } \\
\text { 2x/sem; TC: TF + TA, } \\
\text { dias alternados. }\end{array}$ & $\begin{array}{l}\text { TF: múltiplas séries ( } 40-90 \% \text { de } 1 \mathrm{RM}) \text {. } \\
\text { TA: } 30 \text { a } 60 \text { min em cicloergômetro, } \\
\text { intensidades abaixo do } \mathrm{LV}_{1} \text {, entre } \mathrm{LV}_{1} \text { e } \\
\mathrm{LV}_{2} \text { e acima do } \mathrm{LV}_{2} .\end{array}$ & $\begin{array}{l}\uparrow \text { potência a } 50 \% \text { de } 1 \mathrm{RM}(10 \%)^{* *} ; \\
\uparrow \mathrm{EMG} \text { VL e VM em TF }(26 \text { e } 25 \%)^{* *} \text { e } \\
\text { TC }(33 \text { e } 23 \%)^{* *} \text {; } \text { performance em } \\
\text { testes funcionais em TF e TC. Nenhu- } \\
\text { ma diferença entre TF e TC. }\end{array}$ \\
\hline Karavirta et al. ${ }^{20}$ & $\begin{array}{l}21 \text { sem; TF: } 2 x / \text { sem; TA: } \\
\text { 2x/sem; TC: TF + TA, } \\
\text { dias alternados. }\end{array}$ & $\begin{array}{l}\text { TF: múltiplas séries ( } 40-90 \% \text { de } 1 \mathrm{RM}) \text {. } \\
\text { TA: } 30 \text { a } 60 \text { min em cicloergômetro, } \\
\text { intensidades abaixo do } L_{1} \text {, entre } L V_{1} \text { e } \\
\mathrm{LV}_{2} \text { e acima do } \mathrm{LV}_{2} .\end{array}$ & $\begin{array}{l}\uparrow 1 \mathrm{RM} \text { em TF e TC }(22 \%)^{* * *} \text {. } \uparrow P T \text { em } \\
\text { TF e TC }(14 \text { e } 20 \%)^{*} \text {; } \uparrow \text { EMG VM em TF } \\
(41 \%)^{*} \text { e TC }(28 \%)^{*} \text {. Maior } \uparrow E M G ~ V L \\
\text { em TF do que TC na semana } 10 \text {, mas } \\
\text { sem diferenças na semana } 21 . \uparrow A S T \\
\text { fibras tipo II somente em TF }(16 \%)^{*} \text {. }\end{array}$ \\
\hline Cadore et al..$^{18}$ & $\begin{array}{l}12 \text { sem; TF: } 3 x / \text { sem; TA: } \\
\text { 3x/sem: TC: TF+TA, TA } \\
\text { antes de TF, mesma } \\
\text { sessão. }\end{array}$ & $\begin{array}{l}\text { TF: } 18-20 R M \text { a 6-8RM. TA: } 30 \text { min em } \\
\text { cicloergômetro, } 80 \text { a } 100 \% \text { do } \text { LV }_{2} \text {. }\end{array}$ & $\begin{array}{l}\uparrow \text { ECO neuromuscular dinâmica* so- } \\
\text { mente em TC no RC a 50, } 75 \text { e } 100 \text { W e } \\
\text { em TF e TC no VL a 100W. }\end{array}$ \\
\hline Cadore et al. ${ }^{32}$ & $\begin{array}{l}12 \text { sem; TF: } 3 x / \text { sem; TA: } \\
\text { 3x/sem: TC: TF+TA, TA } \\
\text { antes e depois do TF, } \\
\text { mesma sessão. }\end{array}$ & $\begin{array}{l}\text { TF: } 18-20 \text { RM a 6-8RM. TA: } 30 \text { min em } \\
\text { cicloergômetro, } 80 \text { a } 100 \% \text { do } \text { LV }_{2} \text {. TC } \\
\text { com TF antes do TA (FA) vs. TC com o } \\
\text { TA antes TF (AF). }\end{array}$ & $\begin{array}{l}\text { maior } \uparrow 1 \text { RM em FA do que AF ( } 35 \text { vs. } \\
21 \%)^{* * *} \text {; maior } \uparrow \text { QM em FA do que } \\
\text { em AF ( } 27 \text { vs. } 15 \%)^{* * *} . \uparrow \text { na espessura } \\
\text { do QF semelhante entre os grupos } \\
(9 \%)^{* * *} \text {. }\end{array}$ \\
\hline
\end{tabular}

$\uparrow$, aumento; min, minutos; ECO, economia; 1RM, 1 repetição máxima; PT, pico de torque isométrico; AST, área de secção transversal; $\mathrm{QF}$, quadríceps femoral; VL, vasto lateral; $V M$, vasto medial; $R C$, reto da coxa; sem, semanas; $\mathrm{x} /$ sem, número de treinos por semana; TF, treinamento de força; TA, treinamento aeróbico; TC, treinamento concorrente; EMG, sinal eletromiográfico; $Q M$, qualidade muscular; $L V_{1}$ e $\mathrm{LV}$, primeiro e segundo limiar ventilatório, respectivamente. ${ }^{* \mathrm{P}}<0,05 ;{ }^{* * \mathrm{P}}<0,01 ;{ }^{* * * \mathrm{P}}<0,001 ; \mathrm{NS}$, não significativo. 


\section{CONSIDERAÇ̃̃ES FINAIS}

Baseado nos resultados dos estudos revisados, a melhora na performance neuromuscular decorrente do treinamento de força em idosos aparenta ser semelhante a de indivíduos jovens. Esse aumento na performance da produção de força muscular decorrente do TF pode ser explicado a partir de adaptações neurais e morfológicas. Todos os estudos que investigaram o treino de força em idosos encontraram aumento na força máxima, seja dinâmica ou isométrica. Entretanto, somente os estudos que treinaram algumas séries de forma explosiva encontraram aumento na potência muscular e na taxa de produção de força. Com relação ao treino concorrente, o efeito de interferência nessa população pode ocorrer quando maiores volumes de treinamento semanais são executados, mas a manipulação da ordem dos exercícios pode prevenir esse efeito, já que a execução do TF antes do TA parecer gerar maior aumento na força muscular do que a ordem inversa. Contudo, mesmo havendo um menor desenvolvimento da força muscular com o treino concorrente comparado ao treino de força isolado, ainda assim é recomendável a execução desse tipo de treinamento, já que o mesmo contempla tanto as adaptações neuromusculares quanto cardiorrespiratórias.

\section{AGRADECIMENTOS}

Os autores gostariam de agradecer à CAPES e o CNPq pelo suporte financeiro desse manuscrito.

\section{REFERÊNCIAS BIBLIOGRÁFICAS}

1. Häkkinen K, Kraemer WJ, Pakarinen A, Triplett-Mcbride T, Mcbride JM, Häkkinen A et al. Effect of heavy resistance/power training on maximal strength, muscle morphology, and hormonal patterns in 60-75 year-old men and women. Can J Appl Physiol 2002;27(3):213-31.

2. Izquierdo M, Häkkinen K, Ibañez J, Garrues M, Antón A, Zúniga A, et al. Effects of strength training on muscle power and serum hormones in middle-aged and older men. J Appl Physiol 2001;90(4):1497-507.

3. Slivka D, Raue U, Hollon C, Minchev K, Trappe S. Single muscle fiber adaptations to resistance training in old ( $>80 \mathrm{yr}$ ) men: evidence for limited skeletal muscle plasticity. Am J Physiol Regul Inter Comp Physiol 2008;295(1):R273-80.

4. Raj IS, Bird SR, Westfold BA, Shield AJ. Effects of eccentrically biased versus conventional weight training in older adults. Med Sci Sports Exerc 2011;44(6):1167-76.

5. Häkkinen K, Parakinen A, Kraemer WJ, Häkkinen A, Valkeinen H, Alen M. Selective muscle hypertrophy, changes in EMG and force, and serum hormones during strength training training in older women. J Appl Physiol 2001a;91(2):569-80.

6. Nogueira W, Gentil P, Mello SNM, Oliveira RJ, Bezerra AJC, Bottaro M. Effects of power training on muscle thickness of older men. Int J Sports Med 2009;30(3):200-4.

7. Häkkinen K, Hakkinen A. Neuromuscular adaptations during intensive strength training in middle-aged and elderly males and females. Electromyogr Clin Neurophysiol 1995; 35(3):137-47.

8. Häkkinen K, Kallinen M, Linnamo V, Pastinen UM, Newton RU, Kraemer WJ. Neuromuscular adaptations during bilateral versus unilateral strength training in middle-aged and elderly men and women. Acta Physiol Scand 1996;158(1):77-88.

9. Häkkinen K, Alen M, Kallinen M. Muscle CSA, force production, and activation of leg extensors during isometric and dinamic actions in middle-aged and older people. JAPA 1998;6(3):232-47. 
10. Häkkinen K, Kallinen M, Izquierdo M, Jokelainen K, Lassila H, Mälkiä E, et al. Changes in agonist-antagonist EMG, muscle CSA, and force during strength training in middle-aged and older people. J Appl Physiol 1998;84(4):1341-9.

11. Häkkinen K, Newton RU, Gordon S, Mccornick M, Volek J, Nindl B, et al. Changes in muscle morphology, electromyographic activity and force production characteristics during progressive strength training in young and older men. J Gerontol A Biol Sci Med Sci 1998;53(6):B415-23.

12. Häkkinen K, Alen M, Kallinen M, Newton RU, Kraemer WJ. Neuromuscular adaptation during prolonged strength training, detraining and re-strength-training in middle-aged and elderly people. Eur J Appl Physiol 2000;83(1):51-62.

13. Häkkinen K, Kraemer WJ, Newton RU, Alen M. Changes in electromyografic activity, muscle fibre and force production characteristics during heavy resistance/ power strength training in middle-aged and older men and women. Acta Physiol Scand 2001;171(1):51-62.

14. Brentano MA, Cadore EL, Silva EM, Ambrosini AB, Coertjens M, Petkowiks R, et al. Physiological adaptations to strength and circuit training in postmenopausal women. J Strength Cond Res 2008;22(6):1818-25.

15. Cadore EL, Pinto RS, Lhullier FLR, Correa CS, Alberton CL, Pinto SS, et al. Physiological effects of concurrent training in elderly men. Int J Sports Med 2010;31(10):689-97.

16. Kraemer WJ, Häkkinen K, Newton RU, Nindl BC, Volek JS, Mccormick M,et al. Effects of resistance training on hormonal response patterns in younger vs. older men. J Appl Physiol 1999;87(3):982-92.

17. Kraemer WJ, Patton JF, Gordon SE, Harman EA, Deschenes MR, Reynolds K, et al. Compatibility of high-intensity strength and endurance training on hormonal and skeletal muscle adaptations. J Appl Physiol 1995;78(3):976-89.

18. Cadore EL, Pinto RS, Pinto SS, Alberton CL, Correa CS, Tartaruga MP, et al. Effects of strength, endurance and concurrent training on aerobic power and dynamic neuromuscular economy in elderly men. J Strength Cond Res 2011;25(3):758-66.

19. Izquierdo M, Ibañez J, Häkkinen K, Kraemer WJ, Larrión JL, Gorostiaga EM. Once weekly combined resistance and cardiovascular training in healthy older men. Med Sci Sports Exerc 2004;36(3):435-43.

20. Karavirta L, Häkkinen A, Sillanpää E, Garcia-Lopez D, Kauhanen A, Haapasaari A, et al. Effects of combined endurance and strength training on muscle strength, power and hypertrophy in 40-67-year-old men. Scand J Med Sci Sports 2011;21(3):402-11.

21. Campbell DT, Stanley JC. Experimental and quasi-experimental designs for research. Chicago, IL: Rand McNally, 1966.

22. Bottaro M, Machado SN, Nogueira W, Scales R, Veloso J. Effect of high versus low-velocity resistance training on muscular fitness and functional performance in older men. Eur J Appl Physiol 2007;99(3):257-64.

23. Cannon J, Kay D, Tarpenning KM, Marino FE. Comparative effects of resistance training on peak isometric torque, muscle hypertrophy, voluntary activation and surface EMG between young and elderly women. Clin Physiol Funct Imaging 2007;27(2):91-100.

24. Putman CT, Xu X, Gillies E, Maclean IM, Bell GJ. Effects of strength, endurance and combined training on myosin heavy chain content and fibre-type distribution in humans. Eur J Appl Physiol 2004;92(4-5):376-84.

25. Cadore EL, Pinto RS, Alberton CL, Pinto SS, Lhullier FLR, Tartaruga MP. Neuromuscular economy, strength and endurance in healthy elderly men. J Strength Cond Res 2011;25(4): 997-1003.

26. Sale DG, Jacobs I, Macdougall JD, Garner S. Comparison of two regimens of concurrent strength and endurance training. Med Sci Sports Exerc 1990;22(3):348-56.

27. Bell GJ, Syrotuik D, Socha T, Maclean I, Quinney HA. Effect of strength and endurance training on strength, testosterone, and cortisol. J Strength Cond Res 1997;11(1):57-64. 
28. Cadore EL, Izquierdo M, Dos Santos MG, Martins, JB, Lhullier FL, Pinto RS, et al. Hormonal responses to concurrent strength and endurance training with different exercise orders. J Strength Cond Res 2012; Jan 3 Epub ahead of print.

29. Wood RH, Reyes R, Welsch MA, Favarolo-Sabatier J, Sabatier M, Lee CM, et al. Concurrent cardiovascular and resistance training in healthy older adults. Med Sci Sports Exerc 2001;33(10):1751-8.

30. Mccarthy JP, Pozniak MA, Agre JC. Neuromuscular adaptations to concurrent strength and endurance training. Med Sci Sports Exerc 2002;34(3):511-9.

31. Silva RF, Cadore EL, Kothe G, Guedes M, Alberton CL, Pinto SS et al. Concurrent training with different aerobic exercises. Int J Sports Med 2012; May 4 Epub ahead of print.

32. Cadore EL, Izquierdo M, Alberton CL, Pinto RS, Conceição M, Cunha G et al. Strength prior to endurance intra-session exercise sequence optimizes neuromuscular and cardiovascular gains in elderly men. Exp Gerontol 2012;47(2)164-9.

33. Sillampää E, Häkkinen A, Nyman K, Cheng S, Karavirta L, Laaksonen DE et al. Body composition and fitness during strength and/or endurance training in older men. Med Sci Sports Exerc 2008;40(5):950-8.

34. Sillampää E, Häkkinen A, Punnonen K, Häkkinen K, Laaksonen DE. Effects of strength and endurance training on metabolic risk factors in healthy 40-65-year-old men. Scand J Med Sci Sports 2009;19(6):885-95.

35. Holviala J, Häkkinen A, Karavirta L, Nyman K, Izquierdo M, Gorostiaga EM et al. Effects of combined strength and endurance training on treadmill load carrying walking performance in aging men. J Strength Cond Res 2010;24(6):1584-95.

36. Holviala J, Kraemer WJ, Sillampää E, Karpinen H, Avela J, Kauhanen A et al. Effects of strength, endurance and combined training on muscle strength, walking speed and dynamic balance in aging men. Eur J Appl Physiol 2012;112(4):1135-47.

37. Karavirta L, Tulppo MP, Laaksonen DE, Nyman K, Laukkanen RT, Kinnunen H,et al. Heart rate dynamics after combined endurance and strength training in older men. Med Sci Sports Exerc 2009;41(7):1436-43.

38. Nader GA. Concurrent strength and endurance training: from molecules to man. Med Sci Sports Exerc 2006;38(11):1965-70.

39. Häkkinen K, Alen M, Kraemer WJ, Gorostiaga E, Izquierdo M, Rusko H, et al. Neuromuscular adaptations during concurrent strength and endurance training versus strength training. Eur J Appl Physiol 2003;89(1):42-52.

40. Narici MV, Maganaris C, Reeves N. Myotendinous alterations and effects of resistive loading in old age. Scand J Med Sci Sports 2005;15(6):392-401.
Endereço para correspondência

Eduardo Lusa Cadore

LAPEX, Escola de Educação Física,

UFRGS

Rua: Felizardo, 750

Bairro: Jardim Botânico

CEP: 90690-200 - Porto Alegre, RS.

Brazil

Email: edcadore@yahoo.com.br 